\title{
Society for Cardiovascular Magnetic Resonance perspective on the 2021 AHA/ACC Chest Pain Guidelines
}

\author{
Andrew E. Arai ${ }^{* *}$ (D, Raymond Y. Kwong ${ }^{2}$, Michael Salerno ${ }^{3}$, John P. Greenwood ${ }^{4}$ and Chiara Bucciarelli-Ducci ${ }^{5}$
}

\section{Society for Cardiovascular Magnetic Resonance perspective on the 2021 AHA/ACC Chest Pain Guidelines}

Diagnostic and treatment guidelines serve several important purposes with an overall aim to improve medical care. The 2021 American Heart Association (AHA)/ American College of Cardiology (ACC) Chest Pain Guidelines [1, 2] represent a dramatic evolution from the prior 2012 ACC/AHA Chest Pain Guidelines [3]. For the practitioner that uses or performs cardiovascular magnetic resonance (CMR), the release of new guidelines is an opportunity to reassess what we do, how we do it, and how CMR should be used relative to other imaging modalities. Guidelines translate scientific evidence into recommendations on how to approach specific patient-related conditions. Though representing the "state-of-the-art" at the time of publication, guidelines ultimately represent the opinions of experts in the field and the quality of contemporaneous published literature. Inevitably, not all differences in opinion can be incorporated. The Society for Cardiovascular Magnetic Resonance (SCMR) endorsed the $2021 \mathrm{AHA} / \mathrm{ACC}$ Chest Pain Guidelines as they accurately incorporate 15 indications for CMR and capture a large proportion of what CMR has to offer patients and clinicians in the evaluation of acute and stable chest pain. This document aims to

*Correspondence: andrewarai@icloud.com

1 Private Consultant, Kensington, MD 20895, USA

Full list of author information is available at the end of the article summarize the new 2021 AHA/ACC Chest Pain Guidelines from an SCMR perspective, to highlight the current role for CMR, to identify where knowledge gaps exist, and to describe areas where some CMR expert opinions may differ with the Guidelines. We hope this effort stimulates debate and more importantly stimulates research efforts to refine and expand appropriate CMR indications in future international guidelines.

\section{Indications for CMR in the 2021 AHA/ACC Chest Pain Guidelines}

The 2021 AHA/ACC Chest Pain Guidelines include many recommendations for the use of CMR which are briefly summarized in the next paragraphs and figures. This summary does not include non-CMR recommendations as the full guidelines are published in the Journal of the American College of Cardiology [1] and Circulation [2]. They are also planned to be published later this year in the Journal of Cardiovascular Magnetic Resonance. Reviewing the full Guidelines is necessary to get a detailed appreciation for how the various imaging modalities are 'weighted' in particular recommendations. Recommendation number from the full document is included for reference (Fig. 1).

\section{Low risk coronary artery disease patients}

For low-risk coronary artery disease (CAD) patients, defined as those with a 30-day risk of death or major adverse cardiovascular event $(\mathrm{MACE})<1 \%$, the 2021 AHA/ACC Chest Pain Guidelines provide a class 2a 


\section{Acute Chest Pain}

Recommendations for Patients with Intermediate Risk and No Known CAD

4.1.2.1 4. For intermediate-risk patients with acute chest pain and no known CAD who are eligible for cardiac testing, either exercise ECG, stress echocardiography, stress PET/SPECT MPI, or stress CMR is useful for the diagnosis of myocardial ischemia.

4.1.2.1 7. For intermediate-risk patients with acute chest pain and no known $C A D$, with an inconclusive CCTA, stress imaging (with echocardiography, PET/SPECT MPI, or CMR) can be useful for the diagnosis of myocardial ischemia.

Recommendations for Intermediate-risk Patients with Acute Chest Pain, and Known CAD

4.1.2.2 5. For intermediate-risk patients with acute chest pain and known CAD who have new onset or worsening symptoms, stress imaging (PET/SPECT MPI, CMR, or stress echocardiography) is reasonable.

\section{2a B-NR}

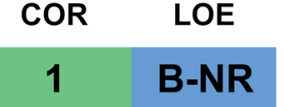

$2 a$ C-EO

\section{Recommendations for High-Risk Patients with Acute Chest Pain}

4.1.3 3. For high-risk patients with acute chest pain who are troponin positive in whom obstructive CAD has been excluded by CCTA or ICA, CMR or echocardiography can be effective in establishing alternative diagnoses.

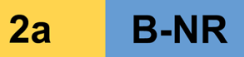

Fig. 1 Acute Chest Pain Recommendations for CMR [1, 2]. CAD coronary artery disease, CCTA coronary artery computed tomography angiography, CMR cardiovascular magnetic resonance, ECG electrocardiogram, ICA invasive coronary angiography, MPI myocardial perfusion imaging, PET positron emission tomography, SPECT single photon emission computed tomography

recommendation indicating that it is reasonable to discharge the patient home without hospitalization or urgent cardiac testing. This is one of the "Top 10 TakeHome Messages" of the updated guidelines.

Reducing imaging indications will reduce imaging costs and the number of cardiac tests that confirm no evidence of significant CAD. While an initial strategy of no-testing is appropriate in these patients, testing remains an option for patients with persistent or worsening symptoms. From a CMR perspective, there is time to schedule appropriate patients in a non-acute setting rather than doing imaging as an emergency procedure.

\section{Intermediate risk patients without known CAD}

The 2021 AHA/ACC Chest Pain Guideline gives a class I indication for stress CMR along with all other stress imaging modalities among intermediate-risk patients without known CAD (4.1.2.1.4). Stress imaging, including CMR, is given a class $2 \mathrm{a}$ recommendation for sequential testing after an inconclusive coronary computed tomography angiographic (CCTA) study (4.1.2.1.7). The 2021 AHA/ACC Chest Pain Guideline puts CMR on par with the other stress imaging modalities regardless of whether or not a patient can exercise or whether or not the electrocardiogram (ECG) is interpretable (Fig. 2).

\section{Intermediate risk patients with known CAD}

For intermediate risk patients with acute chest pain and known CAD, all stress imaging modalities are given a class 2a recommendation (4.1.2.2.5) along with CCTA, in patients with previously known non-obstructive CAD, and without any preference of one modality over the others. This recommendation also brings CMR to the same level as the other non-invasive imaging modalities. Of note, among those with known CAD, exercise testing without imaging is no longer considered an appropriate study.

\section{High-risk patients with acute chest pain}

There is a class 2a recommendation for CMR or echocardiography to establish alternative diagnoses once obstructive CAD has been excluded by CCTA or invasive coronary angiography (ICA). CMR has become well established for detecting other pathologies such is myocardial infarction with no obstructive coronary arteries (MINOCA) (4.2.3.1) or myocarditis which can present acutely in the absence of obstructive CAD (4.1.3.3). Stress CMR could also be used to establish the diagnosis of ischemia in patients with no obstructive coronary arteries (INOCA) or microvascular disease (5.2.2.3).

The 2021 AHA/ACC Chest Pain Guidelines favor computed tomography $(\mathrm{CT})$ as the first choice in the assessment of possible acute aortic syndromes given its faster speed and wider availability. CMR is given a class I recommendation 


\section{Other Scenarios for Acute Chest Pain \\ Recommendations for Acute Chest Pain With Suspected Acute Aortic Syndrome \\ COR LOE \\ 4.2.1 2. In patients with acute chest pain where there is clinical concern for aortic dissection, TEE or CMR should be performed to make the diagnosis

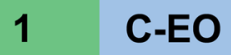
if $\mathrm{CT}$ is contraindicated or unavailable.

\section{Recommendations for Acute Chest Pain With Suspected Myopericarditis}

4.2.3 1. In patients with acute chest pain and myocardial injury who have nonobstructive coronary arteries on anatomic testing, CMR with gadolinium contrast is effective to distinguish myopericarditis from other causes, including myocardial infarction and nonobstructive coronary arteries (MINOCA).

4.2.3 In patients with acute chest pain with suspected acute myopericarditis, CMR is useful if there is diagnostic uncertainty, or to determine the presence and extent of myocardial and pericardial inflammation and fibrosis.

Recommendations for Acute Chest Pain With Valvular Heart Disease (VHD)

4.2.4 In patients presenting with acute chest pain with known or suspected VHD, CMR imaging is reasonable as an alternative to TTE and/or TEE if nondiagnostic.

\section{$1 \quad$ B-NR}

$1 \quad$ B-NR

Fig. 2 Other Scenarios for Acute Chest Pain—Recommendations for CMR [1, 2]. CT computed tomography, MINOCA myocardial infarction with no obstructive coronary arteries, TEE transesophageal echocardiography, TTE transthoracic echocardiography, VHD valvular heart disease. Other abbreviations as in Fig. 1

as an alternative if $\mathrm{CT}$ is contraindicated or unavailable (4.2.1.2).

In patients with possible myopericarditis (4.2.3.2), the new guidelines give CMR a class I recommendation for distinguishing myocarditis from other causes and for assessing myocardial and pericardial inflammation and fibrosis. These recommendations align with increasing community awareness of CMR as the test of choice for an indication often labeled MINOCA. (4.2.3.1).

The 2021 AHA/ACC Chest Pain Guidelines also support a class 2 a recommendation among patients with acute chest pain and known or suspected valvular heart disease if transthoracic echocardiography (TTE) or transesophageal echocardiography (TEE) are not technically adequate for assessing valvular heart disease (4.2.4.3). CMR has the ability to objectively quantify the severity of regurgitant heart lesions (Fig. 3).

Intermediate-high risk patients with stable chest pain and no known CAD

Among intermediate-high risk patients with stable chest pain with no known CAD, CCTA and stress imaging are given class I recommendations (5.1.3.2). Again,
CMR is not differentiated from the other stress imaging modalities.

\section{Stable patients with known CAD}

In patients with known obstructive CAD who have stable chest pain despite optimal therapy, stress imaging receives a class I indication for diagnosing myocardial ischemia, estimating risk of MACE, and guiding therapeutic decision making (5.2.1.5). There is an additional new class 2a indication for both PET and CMR adding quantification of myocardial blood flow reserve (MBFR) to improve diagnostic accuracy and enhance risk stratification (5.2.1.8). This is particularly important given the growing availability of CMR techniques for performing absolute quantification of myocardial blood flow.

In patients with known non-obstructive CAD, stress CMR received a Class 2 a recommendation for assessing INOCA (5.2.2.3). Stress CMR with the addition of quantitative myocardial blood flow assessment is given a class 2 a recommendation for the diagnosis of coronary microvascular dysfunction and for assessing MACE (5.2.3.3). 


\section{Stable Chest Pain}

Recommendations for Intermediate-High Risk Patients With Stable Chest COR LOE Pain and No Known CAD

5.1.3 2. For intermediate-high risk patients with stable chest pain and no known CAD, stress imaging (stress echocardiography, PET/SPECT MPI or CMR) is effective for diagnosis of myocardial ischemia and for estimating risk of MACE.

Sequential or Add-on Testing: What to Do if Index Test Results are Positive or Inconclusive

5.1.3 10. For intermediate-high risk patients with stable chest pain after inconclusive CCTA, stress imaging is reasonable.

\section{2a B-NR}

Recommendations for Patients With Obstructive CAD Who Present With Stable Chest Pain

5.2.1 5. For patients with obstructive CAD who have stable chest pain despite optimal GDMT, stress PET/SPECT MPI, CMR, or echocardiography

1 B-NR is recommended for diagnosis of myocardial ischemia, estimating risk of MACE, and guiding therapeutic decision-making.

5.2.1 8. For patients with obstructive CAD who have stable chest pain symptoms undergoing stress PETMPI or stress CMR, the addition of MBFR is useful to improve diagnosis accuracy and enhance risk stratification.

\section{Recommendations for Patients With Prior CABG Surgery With Stable Chest Pain}

5.2.1.1 2. In patients who have had prior $C A B G$ surgery presenting with stable chest pain who are suspected to have myocardial ischemia, it is reasonable to perform stress imaging or CCTA to evaluate for myocardial ischemia or

2a C-LD graft stenosis or occlusion.

Recommendations for Patients With Known Nonobstructive CAD Presenting With Stable Chest Pain

5.2.2 3. For patients with known extensive nonobstructive CAD with stable chest pain symptoms, stress imaging (PET/SPECT, CMR, or echocardiography) is reasonable for the diagnosis of myocardial ischemia

\section{2a B-NR}

5.2.3 3. For patients with persistent stable chest pain and nonobstructive CAD, stress CMR with the addition of MBFR measurement is reasonable to improve diagnosis of coronary myocardial dysfunction and for estimating

$2 a \quad B-N R$
risk of MACE.

Fig. 3 Stable Chest Pain Guidelines for CMR [1, 2]. INOCA, ischemia with no obstructive coronary arteries; MACE, major adverse cardiovascular event; $M B F R$, myocardial blood flow reserve. Other abbreviations as in Figs. 1 and 2

\section{A case example from the meta-analysis literature (Knuuti et al. [4])}

"In a 55-year old male patient with atypical angina CCTA, single photon emission computed tomography (SPECT), PET and stress CMR can reliably ruleout anatomically significant CAD but stress ECG or stress echocardiography cannot (A). To assess the performance of imaging tests to detect functionally significant CAD (assessed by fractional flow reserve (FFR)) in the same patient (B) one can see that PET and stress CMR results can both rule-out and rulein significant $C A D$ while CCTA can only confidently rule-out if a negative result is documented. ICA and SPECT are not recommended tests in this patient." (Fig. 4) 

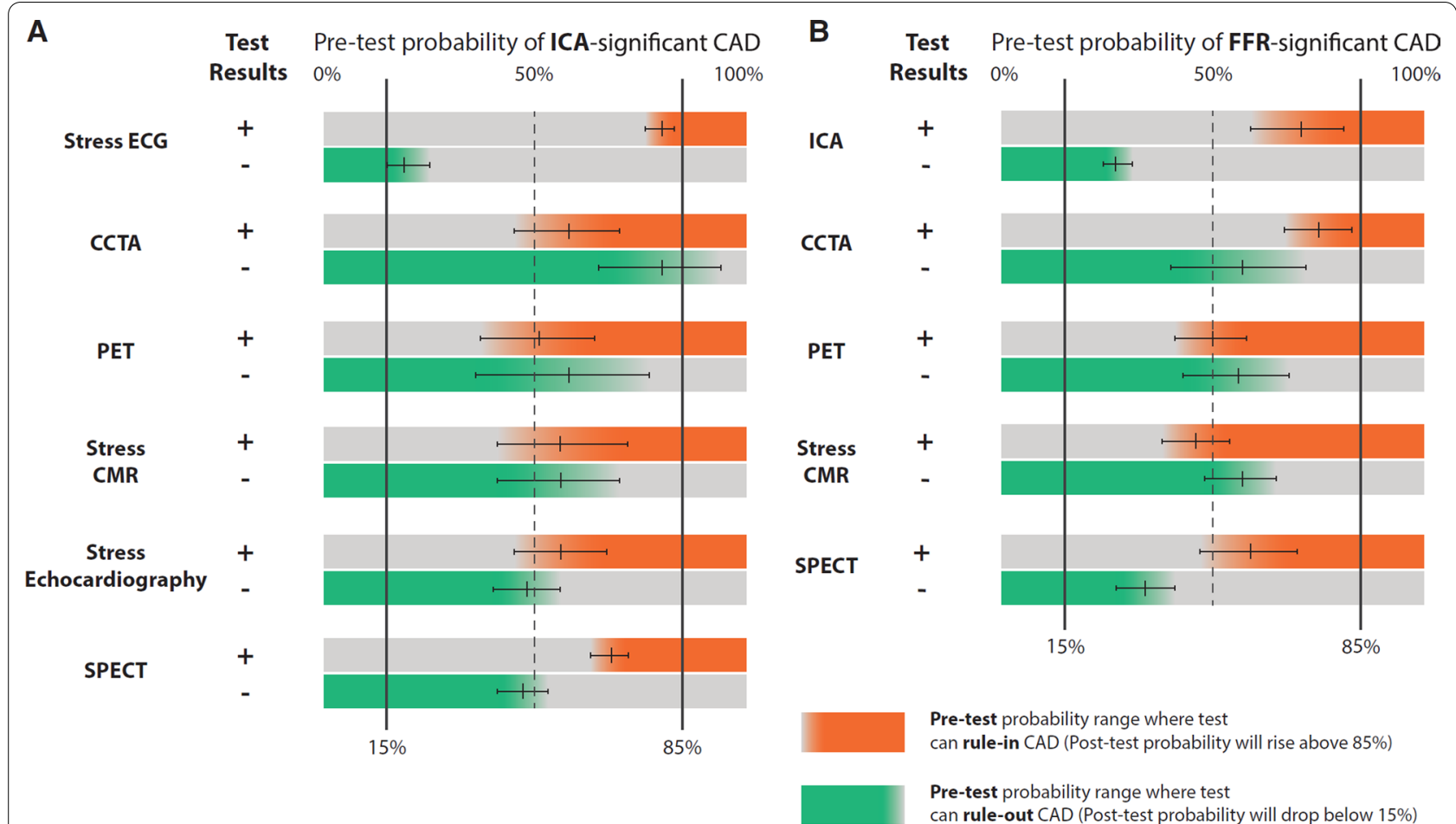

Fig. 4 Ranges of clinical pre-test probability in which each single-positive test will confidently rule-in (in orange) the presence of significant CAD with post-test probability $>85 \%$ or, conversely a negative test will confidently rule-out CAD (in green) with post-test probability $<15 \%$ [4]

\section{Disagreement with some aspects of the guidelines The recommendations of FFR-CT are premature}

The CCTA field recognizes that CCTA (and ICA) cannot confidently rule-in functionally significant $C A D$ and now advocates fractional flow reserve (FFR)-CT to determine functional significance of what is anatomically described as "obstructive CAD" (50-90\% stenosis) and extending further to a $40 \%$ narrowing. A stress CMR may be more cost-effective than FFR-CT or PET in sequential testing. While, CCTA may be the best test to exclude anatomically significant CAD, PET and CMR can effectively rulein and rule-out functionally significant CAD.

The 2021 AHA/ACC Chest Pain Guidelines have 4 recommendations for FFR-CT in acute and chronic stable chest pain syndromes and FFR-CT was broadly described as an instrument for assessing likelihood of ischemia with established robustness for decision-making in lesions of $40-90 \%$ detected on CTA. FFR-CT was displayed in numerous flow charts to represent equivalent class $2 \mathrm{a}$ recommendations compared to any other functional imaging modality.

However, the diagnostic and prognostic utilities of FFR-CT are not as robustly evidenced as any of the stress imaging modalities (stress CMR, SPECT, PET, and stress echocardiography). FFR-CT does not improve the sensitivity of CT, and only modestly improves the specificity of identifying flow-limiting obstructive coronary artery lesions when compared with invasive FFR and functional testing [5-8]. FFR-CT has only limited diagnostic accuracy in detecting hemodynamically significant $\mathrm{CAD}$ in the intermediate range of coronary stenosis of 0.6-0.85 where management decisions are most needed [7]. Studies evaluating FFR-CT have shown inferior incremental diagnostic and prognostic value in comparison to functional testing [7, 9]. Additionally, the recent FORECAST (Fractional Flow Reserve Derived From Computed Tomography Coronary Angiography in the Assessment and Management of Stable Chest Pain) trial of over 1400 patients, while referral to invasive angiography was lower, the use of FFR-CT did not demonstrate any benefits in terms of healthcare costs, cardiovascular outcomes, or quality of life compared to CT-alone [10]. Similarly, the recent RAPID-CT (Rapid Assessment of Potential Ischaemic Heart Disease with CTCA) trial [11] included 1748 patients with intermediate risk with suspected or a provisional diagnosis of acute coronary syndrome randomised to Early CCTA and standard of care compared with standard of care only. The study demonstrated that early CCTA did not alter overall coronary therapeutic interventions or one-year clinical outcomes. 


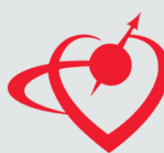

Society for

Cardiovascular

Magnetic

Resonance

\section{Ten Take-Home Messages for} the Evaluation and Diagnosis of Chest Pain from an SCMR Perspective
CMR has 13 indications for the assessment of chest pain.

High sensitivity and High specificity favor CMR and PET.

Emergency assessment of chest pain requires ECG and hs-Troponin

Sex could favor zero radiation CMR over SPECT and $\mathrm{CT}$ considering radiation to the female breast.

Trials are needed to directly compare pathways proposed in Guidelines.
Prognosis is excellent after negative CMR - warranty is better than 2 years per SPINS registry.

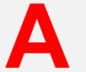

"As Low As Reasonably Achievable" radiation dose points to zero ionizing radiation from CMR \& Echo

INOCA (ischemia) and MINOCA (MI) with no obstructive coronary artery disease are Class 1 indications for CMR.

No urgent imaging test needed in low-risk patients presenting with chest pain.

Stress CMR has Class 1 indications in patients with stable chest pain with or without known CAD

Fig. 5 Ten Take-Home Messages for the Evaluation and Diagnosis of Chest Pain from an SCMR Perspective

Furthermore, there are practical implications regarding FFR-CT. FFR-CT is only feasible in a subset of CCTA cases that are relatively artifact-free and remains highly limited in patients with prior coronary artery stenting, extensive calcification, severe valvular heart disease, sequential luminal lesions or prior coronary artery bypass graft surgery. FFR-CT is currently only available by a single company (HEARTFlow, Redwood City, California, USA). The CPT Category III code used to reimburse FFR-CT is reserved for emerging technologies. Finally, FFR-CT costs 3-times as much as a standard CCTA.

\section{The ischemia imaging modalities were inappropriately compared as a group against anatomical CCTA}

The 2021 AHA/ACC Chest Pain Guidelines put all stress imaging modalities in the same "functional imaging" group for the purpose of comparison against CCTA, as shown in many flow charts and tables. This approach has little clinical basis but also misses critical attributes of different ischemia tests that may be relevant in the management of a vastly diverse patient spectrum. In the contemporary era, there is clear randomised trial evidence for the use of stress CMR in reducing unnecessary ICA referral or coronary revascularization rates, and thus improving patient care, health outcomes and healthcare resource utilization $[12,13]$.

The largest randomized trial to date, PROMISE (PROspective Multicenter Imaging Study for Evaluation of chest pain), prospectively evaluated the utility of an anatomic (CCTA) testing approach in comparison with functional testing (stress imaging and treadmill ECG testing) amongst 193 North American centers in over 10,000 patients [14]. After a median of 2-years follow-up, no differences in adverse cardiac outcomes were observed, while the anatomic approach led to higher downstream utilization of both ICA and coronary revascularization.

The SCOT-HEART (Scottish COmputed Tomography of the HEART) trial conducted within the United Kingdom failed to meet its original primary endpoint, but did observe a late reduction in non-fatal myhocardial infarction (MI) [15]. However, it was a trial of serial testing in one arm (standard care using ETT plus CCTA) versus standard of care (ETT only). This form of layered testing might be expected to produce better outcomes, especially as exercise tolerance testing (ETT) without imaging is well recognized for lower sensitivity and specificity than stress imaging tests. In addition, participants in the SCOT-Heart trial were not systematically treated with optimal medical therapy for primary prevention, a standard of care that has been part of modern cardiology practice for many years.

\section{Definitions of obstructive CAD, anatomically significant $C A D$, and functionally significant $C A D$}

The 2021 AHA/ACC Chest Pain Guidelines do not have clear definitions of "Obstructive CAD", "Anatomically Significant CAD", and "Functionally Significant CAD". These three definitions overlap with each other and contribute to miscommunication among healthcare providers and confuse patients. In general, the 2021 AHA/ACC Chest Pain Guidelines do not systematically differentiate anatomic CAD and functional CAD. This may contribute to an overestimation of the utility of CCTA compared with stress imaging modalities. 
"Obstructive CAD" has been used to describe a 50\% or greater coronary artery diameter stenosis by quantitative coronary angiography (QCA) and is now widely used to describe CCTA findings. However, the majority of $50-70 \%$ stenoses are not severe enough to impair coronary flow reserve [16]. Invasive FFR has been shown in several multi-center trials to provide better outcomes than management by stenosis severity [17-19]. Thus, "functionally significant CAD" represents the subset of coronary artery stenoses that impair flow during vasodilation or increased coronary flow demand. Stress imaging tests are inherently designed to detect abnormal flow reserve detected during exercise or pharmacological stress. We believe the cardiology community should work to clarifying terminology and avoid using words like "obstructive" that suggest a physiological importance to an anatomic stenosis that may or may not impair coronary flow reserve.

\section{Women's health}

The 2021 AHA/ACC Chest Pain Guidelines briefly mention women-specific considerations in text but do not make any formal recommendations that recognize appropriateness or level of evidence. CCTA, SPECT, and PET directly deliver radiation to the breasts. Now that zero ionizing radiation methodologies like CMR can perform as well as CCTA and PET, and are superior to stress echocardiography and SPECT, CMR seems the logical choice for stress perfusion imaging in women if local equipment and expertise is available.

\section{Concluding thoughts and directions for future research}

CMR has matured into a powerful diagnostic tool as evidenced by the wide range of clinical indications recognized in the 2021 AHA/ACC Chest Pain Guidelines and other international guidelines. Figure 5 summarizes 10 Take-Home Messages for the Assessment and Diagnosis of Chest Pain-from a SCMR perspective. CMR practitioners should continue to advocate the importance of the functional significance of CAD.

The CMR community should be proud of the hard work that provided the data leading to multiple Class I and Class 2a Recommendations, which are now finally more aligned with the 2014 ESC Guidelines on Revascularization in which stress CMR features in Class IA recommendations [20]. However, more research is needed regarding the comparative effectiveness of CMR relative to other stress imaging techniques and specifically, CCTA. Randomized clinical trials comparing costs and outcomes of different management strategies will be important.
CMR and PET appear to have superior diagnostic accuracy compared with SPECT and stress echocardiography. The relatively low specificity of CCTA for functionally significant CAD is a weakness. The reliance on FFR-CT to provide a computer-based substitute for a full physiological assessment of CAD may or may not be costeffective over the long haul. CMR researchers should also continue to refine quantitative methods as the current Guidelines are the first to formally recognize the value of quantifying myocardial blood flow reserve by CMR. Studies focused on women will help highlight the role of stress CMR versus other modalities in the diagnosis of CAD as well as other causes of symptoms.

\section{Abbreviations \\ ACC: American College of Cardiology; AHA: American Heart Association; CAD: Coronary artery disease; CCTA: Coronary computed tomography angiography; CMR: Cardiovascular magnetic resonance; CT: Computed tomography; ECG: Electrocardiogram; ETT: Exercise tolerance test; FFR: Fractional flow reserve; ICA: Invasive coronary angiography; INOCA: Ischemia with no obstructive coronary arteries; MACE: Major adverse cardiovascular event; MBFR: Myocar- dial blood flow reserve; MI: Myocardial infarction; MINOCA: Myocardial infarc- tion with no obstructive coronary arteries; MPI: Myocardial perfusion imaging; PET: Positron emission tomography; QCA: Quantitative coronary angiography; SCMR: Society for Cardiovascular Magnetic Resonance; SPECT: Single photon emission computed tomography; TEE: Transesophageal echocardiography; TTE: Transthoracic echocardiography; VHD: Valvular heart disease.}

\section{Authors' contributions}

All authors helped develop the conceptual framework for the paper. AEA prepared the first draft. All authors provided critical review and edits. All authors read and approved the final manuscript.

Funding

SCMR paid licensing fees to reuse previously published figures.

Availability of data and materials

All data and materials are in the public domain and thus available.

\section{Declarations}

Ethics approval and consent to participate

Not applicable.

Consent for publication

All authors consent to publication of this work.

\section{Competing interests}

AEA has licensed software pertaining to perfusion quantification and a patent pending. CBD is the Chief Executive Officer (part-time) of the Society for Cardiovascular Magnetic Resonance (SCMR). Other authors did not identify any competing interests directly related to the material in this editorial.

\section{Author details}

${ }^{1}$ Private Consultant, Kensington, MD 20895, USA. ${ }^{2}$ Brigham and Women's Hospital, Boston, MA, USA. ${ }^{3}$ Stanford University, Palo Alto, CA, USA. ${ }^{4}$ Leeds Institute of Cardiovascular and Metabolic Medicine, University of Leeds, Leeds, UK. ${ }^{5}$ Royal Brompton and Harefield Hospitals, Guys' and St Thomas NHS Hospitals and School of Biomedical Engineering \& Imaging Sciences, King's College London, London, UK.

Received: 27 November 2021 Accepted: 30 November 2021 Published online: 03 January 2022 


\section{References}

1. Gulati M, Levy PD, Mukherjee D, et al. AHA/ACC/ASE/CHEST/SAEM/SCCT/ SCMR guideline for the evaluation and diagnosis of chest pain: a report of the American college of cardiology/American heart association joint committee on clinical practice guidelines. J Am Coll Cardiol. 2021. https:// doi.org/10.1016/j.jacc.2021.07.053.

2. Gulati M, Levy PD, Mukherjee D, et al. AHA/ACC/ASE/CHEST/SAEM/SCCT/ SCMR guideline for the evaluation and diagnosis of chest pain: a report of the American college of cardiology/American heart association joint committee on clinical practice guidelines. Circulation. 2021. https://doi. org/10.1161/CIR.0000000000001029.

3. Fihn SD, Gardin JM, Abrams J, et al. 2012 ACCF/AHA/ACP/AATS/PCNA/ SCAI/STS guideline for the diagnosis and management of patients with stable ischemic heart disease: executive summary. Circulation. 2012;126:3097-137.

4. Knuuti J, Ballo H, Juarez-Orozco LE, et al. The performance of non-invasive tests to rule-in and rule-out significant coronary artery stenosis in patients with stable angina: a meta-analysis focused on post-test disease probability. Eur Heart J. 2018;39:3322-30.

5. Douglas PS, Pontone G, Hlatky MA, et al. Clinical outcomes of fractional flow reserve by computed tomographic angiography-guided diagnostic strategies vs. usual care in patients with suspected coronary artery disease: the prospective longitudinal trial of FFR(CT): outcome and resource impacts study. Eur Heart J. 2015;36:3359-67.

6. Douglas PS, De Bruyne B, Pontone G, et al. 1-Year outcomes of FFRCTguided care in patients with suspected coronary disease: the PLATFORM study. J Am Coll Cardiol. 2016;68:435-45.

7. Driessen RS, Danad I, Stuijfzand WJ, et al. Comparison of coronary computed tomography angiography, fractional flow reserve, and perfusion imaging for ischemia diagnosis. J Am Coll Cardiol. 2019;73:161-73.

8. Cook CM, Petraco R, Shun-Shin MJ, et al. Diagnostic accuracy of computed tomography-derived fractional flow reserve: a systematic review. JAMA Cardiol. 2017:2:803-10.

9. Ahmed Al, Han Y, Al Rifai M, et al. Prognostic value of computed tomography-derived fractional flow reserve comparison with myocardial perfusion imaging. JACC Cardiovasc Imaging. 2021. https://doi.org/10 1016/j.jcmg.2021.09.007.

10. Gray AJ, Roobottom C, Smith JE, et al. Early computed tomography coronary angiography in patients with suspected acute coronary syndrome: randomised controlled trial. BMJ. 2021. https://doi.org/10.1136/bmj. n2106.

11. Curzen N, Nicholas Z, Stuart B, et al. Fractional flow reserve derived from computed tomography coronary angiography in the assessment and management of stable chest pain: the FORECAST randomized trial. Eur Heart J. 2021;42:3844-52.

12. Nagel E, Greenwood JP, McCann GP, et al. Magnetic resonance perfusion or fractional flow reserve in coronary disease. N Engl J Med. 2019;380:2418-28.

13. Greenwood JP, Ripley DP, Berry C, et al. Effect of care guided by cardiovascular magnetic resonance, myocardial perfusion scintigraphy, or NICE guidelines on subsequent unnecessary angiography rates: the CE-MARC 2 randomized clinical trial. JAMA. 2016;316:1051-60.

14. Douglas PS, Hoffmann U, Patel MR, et al. Outcomes of anatomical versus functional testing for coronary artery disease. N Engl J Med. 2015;372:1291-300.

15. Investigators SCOT-HEART, Newby DE, Adamson PD, et al. Coronary CT angiography and 5-year risk of myocardial infarction. N Engl J Med. 2018:379:924-33.

16. Tonino PAL, Fearon WF, De Bruyne B, et al. Angiographic versus functional severity of coronary artery stenoses in the FAME study fractional flow reserve versus angiography in multivessel evaluation. J Am Coll Cardiol. 2010;55(25):2816-21. https://doi.org/10.1016/j.jacc.2009.11.096.

17. De Bruyne B, Pijls NHJ, Kalesan B, et al. fractional flow reserve-guided $\mathrm{PCl}$ versus medical therapy in stable coronary disease. N Engl J Med. 2012;367:991-1001. https://doi.org/10.1056/NEJMoa1205361.

18. Fearon WF, Nishi T, De Bruyne B, et al. Clinical outcomes and costeffectiveness of fractional flow reserve-guided percutaneous coronary intervention in patients with stable coronary artery disease. Circulation. 2018;137(5):480-7.
19. Pijls NHJ, van Schaardenburgh P, Manoharan G, et al. Percutaneous coronary intervention of functionally nonsignificant stenosis: 5-year follow-up of the DEFER study. J Am Coll Cardiol. 2007:49:2105-11.

20. Windecker S, Kolh P, Alfonso F, et al. 2014 ESC/EACTS Guidelines on myocardial revascularization. Eur Heart J. 2014;35:2541-619. https://doi. org/10.1093/eurheartj/ehu278.

\section{Publisher's Note}

Springer Nature remains neutral with regard to jurisdictional claims in published maps and institutional affiliations.
Ready to submit your research? Choose BMC and benefit from:

- fast, convenient online submission

- thorough peer review by experienced researchers in your field

- rapid publication on acceptance

- support for research data, including large and complex data types

- gold Open Access which fosters wider collaboration and increased citations

- maximum visibility for your research: over 100M website views per year

At BMC, research is always in progress.

Learn more biomedcentral.com/submissions 